\title{
Breast cancer risk factors
}

\author{
Marzena Kamińska ${ }^{1}$, Tomasz Ciszewski ${ }^{1}$, Karolina Łopacka-Szatan ${ }^{1}$, Paweł Miotła ${ }^{2}$, Elżbieta Starosławska ${ }^{1}$ \\ ${ }^{1}$ Clinical Oncology Ward, St. John's Cancer Center, Lublin, Poland \\ $2^{2} 2^{\text {nd }}$ Department of Gynecology, Medical University of Lublin, Poland
}

\begin{abstract}
Breast cancer is the most frequently diagnosed neoplastic disease in women around menopause often leading to a significant reduction of these women's ability to function normally in everyday life. The increased breast cancer incidence observed in epidemiological studies in a group of women actively participating in social and professional life implicates the necessity of conducting multidirectional studies in order to identify risk factors associated with the occurrence of this type of neoplasm. Taking the possibility of influencing the neoplastic transformation process in individuals as a criterion, all the risk factors initiating the process can be divided into two groups. The first group would include inherent factors such as age, sex, race, genetic makeup promoting familial occurrence of the neoplastic disease or the occurrence of benign proliferative lesions of the mammary gland. They all constitute independent parameters and do not undergo simple modification in the course of an individual's life. The second group would include extrinsic factors conditioned by lifestyle, diet or long-term medical intervention such as using oral hormonal contraceptives or hormonal replacement therapy and their influence on the neoplastic process may be modified to a certain degree. Identification of modifiable factors may contribute to development of prevention strategies decreasing breast cancer incidence.
\end{abstract}

Key words: breast cancer, risk factors, epidemiology.

\section{Introduction}

Breast cancer is the most frequently diagnosed neoplastic disease in women around menopause often leading to a significant reduction of these women's ability to function normally in everyday life. According to 2010 epidemiological data for the Polish population published on the National Cancer Registry web page (www.onkologia.org.pl), the number of new breast cancer cases was 15 784, which constituted about 22\% of all diagnosed cancers. The age distribution within the population of breast cancer patients is also quite characteristic. Eighty percent of these cancers are diagnosed in women aged 50 and more. Moreover, according to epidemiological data, $50 \%$ of breast cancers occur in women aged from 50 to 69 years. What is especially worrying, within the last two decades we have observed an over 2-fold increase in the occurrence of breast cancer in the Polish population, which, unfortunately, has not been correlated with a significant improvement in treatment outcomes. As it was presented in the analysis by the $\mathrm{Na}$ tional Cancer Registry, 5-year survival was observed in $75 \%$ of patients diagnosed in the years 2000-2002 and in $77.2 \%$ of patients diagnosed in the years 2003-2005.

The increased breast cancer incidence observed in epidemiological studies in a group of women actively participating in social and professional life implicates the necessity of conducting multidirectional studies in order to identify risk factors associated with the occurrence of this type of neoplasm. Intensive studies conducted over the past several years showed that 20-30\% of newly diagnosed breast cancer cases may be associated with the occurrence of various risk factors actively initiating or modifying the process of neoplastic transformation of breast cells. The most important ones are age over 40 , history of mammary gland diseases, history of cancer in first-degree relatives, early menarche and late childbearing (after 35 years of age), woman's age at menopause and Caucasian race [1]. Despite the identification of many factors increasing the risk of breast cancer occurrence, in $75-80 \%$ of women no risk factor is found [1]. The above-mentioned factors do not exhaust the list of possible factors. They only illustrate multidirectionality of the epidemiological, molecular and clinical studies that have been conducted. Taking the possibility of influencing the neoplastic transformation process in individuals as a criterion, all the risk factors initiating the process can be divided into two groups. The first group would include inherent factors such as age, sex, race, genetic makeup promoting familial occurrence of neoplastic disease or the occurrence of benign proliferative lesions of the mammary 
gland. They all constitute independent parameters and do not undergo simple modification in the course of an individual's life. The second group would include extrinsic factors conditioned by lifestyle, diet or longterm medical intervention and their influence on the neoplastic process may be modified to a certain degree. However, it should be stressed that despite intensive studies and examinations, it is difficult to unequivocally explain the etiopathogenesis of the disease.

\section{Intrinsic factors conditioning breast cancer occurrence}

The first basic factor in this group is the patient's age at the moment of diagnosis of neoplastic disease. As it was mentioned above, breast cancer is most frequently found in women around menopause. It is significantly less frequently found in women below 45 years of age. The analysis of morbidity coefficients for the Polish population has indicated a linear increase in the group of women aged between 40 and 59 years, then it reaches a plateau with a slight decreasing tendency in women aged 70 and older. A very interesting correlation can be observed between the age when neoplastic disease is diagnosed and the expression of the estrogen receptor found in the examined tumor tissue. Neoplasms showing estrogen receptor overexpression ER (+) are characterized by a frequency increasing with age as opposed to ER (-) tumors, which occur more frequently up to 50 years of age and then reach a plateau. This phenomenon explains an increased percentage of ER (+) tumors diagnosed in women after menopause [2].

Analyzing the structure of occurrence of breast cancer in relation to sex, we can firmly state that the neoplasm is diagnosed predominantly in women and only sporadically in men in whom it constitutes less than $1 \%$ of all diagnosed breast cancers [3]. Despite the incidental occurrence of this type of neoplasm in men, epidemiological data analysis has shown a clear increase in the occurrence of breast cancer in men over the last three decades $[4,5]$. This phenomenon is difficult to explain, which is probably a result of the civilization progress ultimately leading to excessive obesity and longer life expectancy in men [4]. On the basis of literature data it is hard to unequivocally describe the disease course and prognosis in this group of patients, which, as it seems, could be a result of a higher degree of advancement of the basic neoplastic process at the moment of diagnosis, coexistence of diseases associated with old age or more aggressive course of the neoplastic disease [2]. Some other studies, however, did not reveal increased mortality due to breast cancer in relation to patients' sex [6, 7]. This variation was initially explained by differences in the molecular mechanism underlying neoplastic transformation of mammary gland cells between women and men. However, numerous studies conducted within the last two decades have not confirmed this hypothesis. What is more, due to significant homology of the neoplastic processes in both sexes, it is nowadays suggested that the same treatment methods should be applied in women and men diagnosed with breast cancer [2]. Detailed histopathological analysis of archive tissue material obtained from male patients diagnosed with breast cancer showed a significantly, over $80 \%$, higher level of overexpression of estrogen and progesterone receptors than the one observed in material obtained from women with breast cancer. This overexpression is very often strictly correlated with an overexpression of bcl-2 protein [8]. However, it is postulated that despite such a large percentage of ER (+) tumors, their response to tamoxifen is weaker and the receptor status is not associated with better prognosis $[2,9]$. Another very interesting phenomenon is a small percentage of tumors with HER-2 (human epidermal growth factor receptor 2) super-expression in this group and reported lack of the associated prognostic value $[10,11]$. Other risk factors leading to the occurrence of breast cancer in both sexes include an increased estrogens level due to obesity, testicles hormonal function impairment or Klinefelter syndrome [8].

Race is a very important intrinsic factor elevating the risk of occurrence of breast cancer. As it was demonstrated in the study of Ban et al. [2], who analyzed data included in SEER (Surveillance, Epidemiology, and End Results) database, the frequency of occurrence of breast cancer in Caucasian women is about 127.4 in 100000 individuals. The indicators of mortality and 5 -year survival are $12.3 \%$ and $90.4 \%$, respectively. In the case of Blacks, the frequency of occurrence of the neoplasm is about 121.4 in 100000 individuals and the indicators of mortality and 5-year survival are $18.2 \%$ and $78.6 \%$, respectively. The increased mortality due to breast cancer as well as a lower number of women surviving over 5 years from the moment of diagnosis is a result of a higher level of progression of the disease at the moment of initiating anti-neoplastic treatment and a high percentage of tumors with no receptor expression (triple negative tumors) [2]. It is worthwhile noticing that there is a much lower incidence of breast cancer among Hispanics, however, in their case, the disease is diagnosed at a younger age and often shows lack of expression of the estrogen or progesterone receptor as well as super-expression of HER2 [2].

Another intrinsic factor conditioning the occurrence of breast cancer is the familial susceptibility to this type of neoplasm. Intensive studies have been conducted in the recent decades, which led to identification of genes whose function disorder is associated with an increased risk of occurrence of malignant breast or ovarian cancer. The most important are genes BRCA1 and BRCA2 (breast cancer susceptibility 1 and 2) fulfilling the function of tumor suppressor genes in a cell. The occurrence 
of changes in the coding sequence may lead to the development of hereditary syndromes called HBC-SS (Hereditary Breast Cancer Site Specific) or HBOC (Hereditary Breast Ovarian Cancer) syndrome, which manifest themselves in the form of breast and/or ovarian cancer. The correlation of epidemiologic and population studies has allowed for the estimation of the number of familial breast and/or ovarian cancer cases. They constitute about $10 \%$ of all newly diagnosed neoplasms of these organs [12]. Identification of mutations in BRCA1 or BRCA2 genes is associated with an increased risk of occurrence of breast and/or ovarian cancer in $65 \%$ or $45 \%$ of mutation carriers, respectively, depending on the mutation type [13]. The hereditary syndromes caused by BRCA1 or BRCA2 gene mutations are associated with several clinical symptoms, which may require a complex molecular analysis in the patient. The first, basic criterion is early age of breast and/or ovarian cancer occurrence, usually in relatively young people below 45 years of age. The second criterion that is taken into account is the identification of familial aggregation of breast and/or ovarian cancer cases in first- and seconddegree relatives on a pedigree [14]. Lack of the abovedescribed aggregation, in the presence of other clinical circumstances, cannot prevent doctors from performing molecular diagnostics in such patients. As it was demonstrated in molecular screening of an unselected population of breast cancer patients, in $35 \%$ to $50 \%$ of the patients who were BRCA mutation carriers, no familial history was found [2]. Due to this fact, the leading medical societies such as ASCO (American Society of Clinical Oncology), ESMO (European Society for Medical Oncology), NCCN (The National Comprehensive Cancer Network) or NICE (The National Institute for Health and Care Excellence) have drafted guidelines concerning molecular diagnostics, defining a group of patients who should undergo examination, in whom there is the highest risk of occurrence of mutations in BRCA1 or $B R C A 2$ genes. The guidelines differ slightly as they have to consider genetic variations of different populations as well as socioeconomic conditions. However, they all assume the necessity of performing genetic testing to evaluate the presence of $B R C A 1$ and/or BRCA2 mutations in patients diagnosed with breast cancer by the age of 35 . The examination should also be performed in all patients diagnosed with breast cancer by the age of 40 whose tumor was characterized by lack of estrogen, progesterone and HER2 receptor overexpression (triple negative patients). Genetic consultation is also recommended to patients with bilateral breast cancer and family history of breast and/or ovarian cancer, especially if the tumor occurred by the age of 50, as well as in men diagnosed with breast cancer [15]. The indication for the examination of mutations in BRCA1 and $B R C A 2$ genes may also be a family history of prostate cancer at an early age, pancreatic cancer or malignant melanoma [16]. Nevertheless, despite meeting the criteria qualifying patients for molecular examination, in over $90 \%$ of tested patients no mutations are found in $B R C A 1$ or BRCA2 genes, which does not exclude, however, the familial background of the neoplastic disease [17]. One of the reasons for this outcome may be the scope of mutations analysis on a molecular level, which does not always fully reflect the "mutation status" of a given population or an individual patient. It most frequently results from economic considerations limiting the analysis to the most frequent mutations in a given population, which leads to overlooking mutations occurring in a given population in only a small percentage of cases but still disrupting the functions of BRCA1 or $B R C A 2$ genes. The quality of the performed molecular evaluation is also strongly influenced by the selection of the analyzed genes resulting from the quality of the pedigree as well as prioritizing the information of neoplastic diseases over the one without any direct connection with the initial diagnosis. Omitting pedigree information, apparently not associated with the basic disease, which may suggest the existence of other hereditary processes may direct the doctor's attention to conclusions excluding the analysis of mutations of other genes that are significantly involved in the neoplastic transformation such as CHEK2 (checkpoint kinase 2), PTEN (phosphatase and tensin homolog), PALB2 (partner and localizer of BRCA2), RAD51C (S. cerevisiae, homolog of, C), $\mathrm{CDH} 1$ (cadherin 1) or genes determining Lynch syndrome [18]. Analyzing the function of genes in the process of development of familial breast and/ or ovarian cancers, one must remember that it can be to a certain degree modified under the influence of environmental factors or result from single nucleotide polymorphisms [19].

Also the role of natural hormonal changes taking place in the period of maturation and their influence on the risk of occurrence of breast cancer in adult life is very interesting. As it was pointed out in a work of Hsieh et al., early menarche is associated with a higher risk of occurrence of breast cancer due to a longer period of exposure to estrogens activity [20]. Each delay of menarche by 2 years reduces the risk of occurrence of breast cancer by $10 \%$. What is interesting, in women in whom menarche occurred before the age of 12, estrogen expression in each menstrual cycle is higher than in women who had menarche after the age of 13 [21]. There is a slightly different correlation between the risk of occurrence of breast cancer and age of the first successful pregnancy. Early pregnancy and due date delivery has a protective value and is associated with a lower risk of breast cancer. This correlation mostly concerns women with hormone dependent (ER positive) breast cancer diagnosed in the postmenopausal period [2]. Similar protective activity, decreasing the risk of occurrence of breast cancer is provided by prolonged breast- 
feeding [2]. As it is estimated on the basis of epidemiologic observations, each year of breastfeeding reduces the risk of neoplastic disease by $4.3 \%$ [22]. Unfortunately, this effect has not been observed in European countries, which is most often connected with cultural habits and drive for quick return to professional activity, thus shortening the period of breastfeeding. The correlation of the above-described observations with the presence of mutations in BRCA1 and BRCA2 genes showed a $32 \%$ breast cancer risk reduction in $B R C A 1$ mutation carriers who breastfed their children for a year or longer. However, a similar phenomenon has not been observed in carriers of BRCA2 gene mutation [2]. Moreover, in breastfeeding women, in whom breast cancer does occur, tumors with super-expression of the estrogen receptor occur three times more frequently than in women who have never breastfed. This observation is hard to explain. It seems that breastfeeding facilitates correct differentiation of epithelial cells of the lactiferous ducts thanks to which they are less susceptible to the activity of mutagenic factors and disorders on the DNA (deoxyribonucleic acid) level, which can occur during the cell replication process [2]. Also the age of menopause is not irrelevant to the risk of occurrence of breast cancer. A literature review clearly demonstrates that each year of continuing menstruation increases the risk of breast cancer by $3 \%$. This observation is also confirmed by a $50 \%$ reduction in the breast cancer risk in women who underwent ovariectomy by the age of 40. This phenomenon is especially visible in the group of carriers of BRCA1 or BRCA2 mutations [2].

When talking about intrinsic factors one must not forget about proliferative lesions of benign character occurring in mammary glands. They are benign, however, they can significantly increase the risk of occurrence of malignant lesions. Based on the epidemiologic data, in the group of women diagnosed with benign proliferative lesions of the mammary glands with cellular atypia and familial aggregation of breast neoplasms in first- and second-degree relatives there was an 11-fold increase in the breast cancer risk compared to women without cellular atypia and family history of cancer [23].

\section{Extrinsic factors conditioning breast cancer occurrence}

One of basic groups of extrinsic factors modifying the risk of breast cancer occurrence includes dietary habits, which may lead to obesity occurring especially frequently in populations of developed countries. Eating products that are rich in fat, leading to excess weight or obesity, as well as processed products containing a range of chemical substances, used to enhance flavor or preserve food, may be a factor promoting the neoplastic transformation process in mammary gland cells [24]. This correlation, especially when observed in women in the postmenopausal period, is associated with an increased risk of occurrence of breast cancer without an overexpression of estrogen, progesterone or HER2 receptors [25]. The above-described observations have been confirmed by Saxe et al. [26]. The authors showed that using low-fat diet in a group of women after menopause, who were treated for neoplastic disease, significantly decreased the risk of neoplasm relapse after the primary surgical procedure. The above-described observations also provide the basis for recommending physical activity to a group of obese women with breast cancer after the primary surgical procedure. The studies proved that regular physical activity, with a frequency of 3-5 times a week reduces the risk of breast cancer occurrence by $20-40 \%$, reinforces the immunological system, improves general fitness and the quality of life [27].

A significant role in the modification of the risk of occurrence of neoplastic disease is played by dietary habits typical for a given age group or population. Eating products containing a large amount of anti-oxidants or rich in vitamin D may lead to a $20 \%$ reduction in the relative risk of breast cancer $[26,28]$. The risk may increase as a result of drinking even a small amount of alcohol as it affects estrogen metabolism in the liver [29].

\section{Endogenous sex hormone blood concentration, hormonal therapy and breast cancer risk}

A high endogenous estrogen level is a well-defined risk factor contributing to a higher incidence of breast cancer. The analysis of prospective studies confirmed a strong association between increasing concentrations of sex hormones (total estradiol, free estradiol, estrone, estrone sulfate, androstenedione, dehydroepiandrosterone, dehydroepiandrosterone sulfate and testosterone) in postmenopausal women and a higher breast cancer risk [30]. The significance of these data among premenopausal women is less clear partially because of a cycle-dependent variability of the hormone concentration in this group. Available studies on large cohorts of observed participants suggest that levels of circulating estrogens and androgens may also play an important role in the etiology of premenopausal breast cancer [31, 32]. The results of the Nurses' Health Study II [33] support another thesis that premenopausal levels of estrogens and androgens may be important for the development of postmenopausal disease. The influence of serum hormone levels on the risk of development of different breast cancer subtypes according to the receptor status was studied by Farhat et al. [34]. The analysis revealed that higher serum levels of bioavailable testosterone may be associated with lower risks of ER-negative breast cancer. On the other hand, both testosterone and estradiol were correlated with an 
increased risk of ER-positive disease in postmenopausal women.

Exemestane, anastrozole and letrozole are aromatase inhibitors (Als) that are known to inhibit the enzyme responsible for the conversion of androgens to estrogens and thus these drugs decrease blood estrogen levels in postmenopausal women. Taken together the above data prompted numerous studies evaluating Als to lower the risk of breast cancer. The NCIC CTG MAP.3 trial was a prospective double-blind trial that investigated the role of exemestane in reducing the incidence of invasive breast cancer in postmenopausal women who were determined to be at increased risk. The trial revealed a significant $65 \%$ relative reduction in the annual incidence of invasive breast cancer among the investigated drug group with no statistically significant differences in the incidence of serious adverse events between groups [35]. Another phase III trial that evaluated the Als in postmenopausal women at high risk of breast cancer was IBIS-II in which anastrozole was associated with a $53 \%$ reduction in the incidence of invasive breast cancer and DCIS (ductal carcinoma in situ) when compared with placebo - the effect was significant in ER positive disease [36]. Current ASCO clinical practice guidelines recommend exemestane as an alternative option to tamoxifen or raloxifene to reduce the risk of invasive breast cancer, specifically ER-positive in postmenopausal women at increased risk of breast cancer or with LCIS (lobular carcinoma in situ) or atypical hyperplasia [37]. This indication was not accepted by the European Medicines Agency.

The relationship between oral contraception (OC) and the risk of breast cancer still remains controversial. Some studies have suggested that using oral hormonal contraceptives increases the risk of breast cancer by $24 \%$ compared to women who have never used them [2], and the largest incidence increase is observed in the course of using the contraception. On the other hand, the reanalysis of epidemiological studies showed a little or even no association between the risk of breast cancer and oral contraception [38]. The differences between analyses could be also associated with changes in formulations of oral contraceptives during past decades. Moreover, different oral contraceptive formulations could lead to different risks of breast cancer. It was also described that the risk of occurrence of breast cancer drops significantly 10 years after discontinuing hormonal therapy and it is not dependent on the duration of their usage [39]. In the 1990s, the Collaborative Group on Hormonal Factors in Breast Cancer confirmed no significant risk of breast cancer diagnosed 10 or more years after stopping OC (RR 1.01). Moreover, the cancers diagnosed in women who had used combined OC were less advanced clinically compared to never users [40]. In the large, prospective study (116 $608 \mathrm{fe}$ males and 1246967 person-years of follow-up) was found that only one formulation of oral contraception was related to an increase in the risk of breast cancer in comparison with never OC users - triphasic ethinyl estradiol combined with levonorgestrel (RR 3.05). It should be underlined that non-triphasic formulations with levonorgestrel were not associated with increasing the risk of breast cancer [41]. Other recent evidence suggests that triphasic OC formulation levonorgestrel is not associated with an increased risk of breast cancer. Further analyses did not show any significantly increase in the breast cancer risk among women aged 35 to 64 (current or former OC users). The relative risk was 1.0 for women who were currently using OC and 0.9 for using in the past. The analysis highlights the lack of association between taking $O C$ and an increased risk of breast cancer in women with a family history of this cancer [39]. In another multicenter, population-based study, women were asked about OC formulation used in the past. The most common used formulations were monophasic (100 $\mathrm{mg}$ mestranol/1.0 mg ethynodiol diacetate; $35 \mu \mathrm{g}$ ethinyl estradiol/0.5 mg norethindrone; $35 \mu \mathrm{g}$ ethinyl estradiol/1.0 mg norethindrone; $50 \mu \mathrm{g}$ mestranol/1.0 mg norethindrone; $80 \mu \mathrm{g}$ mestranol/ $1.0 \mathrm{mg}$ norethindrone; $100 \mu \mathrm{g}$ mestranol/2.0 mg norethindrone; $100 \mu \mathrm{g}$ mestranol/2.5 mg norethindrone; $30 \mu \mathrm{g}$ ethinyl estradiol/0.3 mg norgestrel; $50 \mu \mathrm{g}$ ethinyl estradiol/0.5 mg norgestrel) and only one was multiphasic (35 $\mu$ g ethinyl estradiol/0.5 mg (one week)/0.75 $\mathrm{mg}$ (one week)/1.0 mg (one week) norethindrone). Based on multivariable analyses the researchers did not observe any significant correlation between OC formulation and increasing the breast cancer risk [42]. The retrospective analysis of a population-based study (5113 breast cancer cases and 20452 controls) did not show any association between use of levonorgestrel releasing intrauterine devices (IUD) and the increase in breast cancer. The recent evidence also excluded that levonorgestrel IUD could have any promotional effect on tumor growth [43]. Very interesting conclusions were obtained in a study in which a lower degree of neoplastic disease progression at the time of diagnosis was observed in women using hormonal contraceptives compared to women who had never used them [40].

Using oral hormonal menopause therapy is associated with an increase in the breast cancer risk, which was correlated with the length of therapy, $15 \%$ in the case of 5 years and $34 \%$ in the case of 10 years [2]. The risk of occurrence of breast cancer is additionally enhanced by the use of two-component oral menopausal hormone therapy containing estrogen and progesterone (especially conjugated equine estrogens - CEE and medroxyprogesterone acetate - MPA) [44]. It should be noticed that the type of progestin may be a risk factor for breast cancer. Use of a synthetic medroxyprogesterone acetate may increase the number of cell division in 
mammary tissue and may cause greater proliferation of abnormal cells. Some authors observed that intake of natural progesterone did not result in the increase in the breast cancer risk however, further long-term studies are needed to clarify this hypothesis [45]. The impact of androgens on promoting and development of breast cancer was unclear for many years. The risk of breast cancer associated with use of menopausal hormone therapy containing testosterone was assessed in the Nurses' Health Study (24 years of follow-up; 1359323 person-years). It was found in this study that the risk of breast cancer was nearly 2.5 -fold greater among current users of estrogen plus testosterone therapies compared to never users of hormonal therapy. Based on results it was calculated that the risk of breast cancer associated with the current use of estrogen and testosterone therapy was significantly greater compared with estrogenonly and slightly greater than estrogen-progestin therapy (EPT). The increase in the breast cancer risk was $17 \%$ per year of taking HT containing testosterone [46]. One of the observations during the Women's Health Initiative Study (WHI) was the fact that menopausal hormone therapy had a delayed effect on the incidence of breast cancer - during the first 2 years of observation followup, the incidence was reduced even by $41 \%$ and it could be associated with increasing of density of breast tissue and decreasing the sensitivity of mammography. That is why, small tumors could be undiagnosed in the early stages and they were detected after two years in an advanced grade [47]. Based on WHI trial results it was shown that the use of an EPT combination is associated with an increase in breast density of $4.9 \%$ after two years. Unopposed estrogen therapy (ET) increased by $2.9 \%$ the density of breast tissue. Moreover, use of EPT led to an increasing number of abnormal results of mammography and the need for more invasive diagnostic method - breast biopsy [48, 49].

Estrogen-progestin therapy is associated with an increase in percent mammographic density (PMD) - the proportion of the total breast area occupied by dense tissue. There is a relationship between changes in PMD and the use of exogenous hormones. The use of EPT is directly associated with extensive percent mammographic density and led to an increase in PMD by $3-5 \%$ and the estrogen alone therapy increased PMD by $1.2 \%$ [48]. On the other hand, use of an anti-estrogenic drugs (raloxifene, tamoxifen or aromatase inhibitors) results in decreasing PMD, even by $13.7 \%$ over 4.5 years. The risk of breast cancer was reduced by $52 \%$ in patients who observed a decrease in PMD of over $10 \%$. It should be noticed that use of tibolone did not change percent mammographic density. Histopathological analysis has shown that extensive PMD is associated with a greater number of cells and total nuclear area (including epithelial and non-epithelial cells) and an increased amount of collagen and glandular structures [50].
Systemic menopausal hormone therapy should be avoided in women who have gene mutations (BRCA1, $B R C A 2)$ or first-degree relatives with breast cancer or atypical findings in breast biopsy. Use of EPT for less than three years probably does not increase the breast cancer risk significantly but it can impede the detection the early stages of breast cancer [51].

\section{Disclosure}

Authors report no conflict of interest.

\section{References}

1. Bucholc M, Łepecka-Klusek C, Pilewska A, et al. Ryzyko zachorowania na raka piersi w opinii kobiet. Ginekol Pol 2001; 72: 1460-1456.

2. Ban KA, Godellas CV. Epidemiology of breast cancer. Surg Oncol Clin N Am 2014; 23: 409-422.

3. Gnerlich JL, Deshpande AD, Jeffe DB, et al. Poorer survival outcomes for male breast cancer compared with female breast cancer may be attributable to in-stage migration. Ann Surg Oncol 2011; 18: 1837-1844.

4. Speirs V, Shaaban AM. The rising incidence of male breast cancer. Breast Cancer Res Treatment 2009; 115: 429-430.

5. Stang A, Thomssen C. Decline in breast cancer incidence in the United States: what about male breast cancer? Breast Cancer Res Treatment 2008; 112: 595-596.

6. Thalib L, Hall P. Survival of male breast cancer patients: populationbased cohort study. Cancer Sci 2009; 100: 292-295.

7. Hill TD, Khamis HJ, Tyczynski JE, et al. Comparison of male and female breast cancer incidence trends, tumor characteristics, and survival. Ann Epidemiol 2005; 15: 773-780.

8. Weiss JR, Moysich KB, Swede H. Epidemiology of male breast cancer. Cancer Epidemiol Biomarkers Prev 2005; 14: 20-26.

9. Giordano SH, Perkins GH, Broglio K, et al. Adjuvant systemic therapy for male breast carcinoma. Cancer 2005; 104: 2359-2364.

10. Avisar E, McParland E, Dicostanzo D, et al. Prognostic factors in nodenegative male breast cancer. Clin Breast Cancer 2006; 7: 331-335.

11. Onami S, Ozaki M, Mortimer JE, et al. Male breast cancer: an update in diagnosis, treatment, and molecular profiling. Maturitas 2010; 65: 308-314.

12. Francken $A B$, Schouten $P C$, Bleiker $E$, et al. Breast cancer in women at high risk: the role of rapid genetic testing for BRCA1 and -2 mutations and the consequences for treatment strategies. Breast 2013; 22: 561-568.

13. Antoniou A, Pharoah PD, Narod S, et al. Average risks of breast and ovarian cancer associated with BRCA1 or BRCA2 mutations detected in case Series unselected for family history: a combined analysis of 22 studies. Am J Hum Genet 2003; 72: 1117-1130.

14. Mohamad HB, Apffelstaedt JP. Counseling for male BRCA mutation carriers: a review. Breast 2008; 17: 441-450.

15. Young SR, Pilarski RT, Donenberg T, et al. The prevalence of BRCA1 mutations among young women with triple-negative breast cancer. BMC Cancer 2009; 9: 86.

16. Oosterwijk JC, de Vries J, Mourits MJ, et al. Genetic testing and familial implications in breast-ovarian cancer families. Maturitas 2014; 78: 252-257.

17. Couch FJ, Nathanson KL, Offit K. Two decades after BRCA: setting paradigms in personalized cancer care and prevention. Science 2014; 6178: 1466-1470.

18. Mavaddat N, Antoniou AC, Easton DF, et al. Genetic susceptibility to breast cancer. Mol Oncol 2010; 3: 174-191.

19. Antoniou AC, Beesley J, McGuffog L, et al. Common breast cancer susceptibility allels and the risk of breast cancer for BRCA1 and BRCA2 mutation carriers: implication for risk prediction. Cancer Res 2010; 23: 9742-9754.

20. Hsieh CC, Trichopoulos D, Katsouyanni K, et al. Age at menarche, age at menopause, height and obesity as risk factors for breast cancer: as- 
sociations and interactions in an international case-control study. Int J Cancer 1990; 46: 796-800.

21. Apter D, Reinila M, Vihko R. Some endocrine characteristics of early menarche, a risk factor for breast cancer, are preserved into adulthood. Int J Cancer 1989; 44: 783-787.

22. Collaborative Group on Hormonal Factors in Breast Cancer. Breast cancer and breastfeeding: collaborative reanalysis of individual data from 47 epidemiological studies in 30 countries, including 50302 women with breast cancer and 96973 women without the disease. Lancet 2002; 360: 187-195.

23. Hartmann LC, Sellers TA, Frost MH, et al. Benign breast disease and the risk of breast cancer. N Engl J Med 2005; 353: 229-237.

24. Konopka P. Rak, układ odpornościowy a odżywianie. Poradnik dla pacjentów. MedPharm, Wrocław 2009.

25. Thiébaut AC, Kipnis V, Chang SC, et al. Dietary fat and postmenopausal invasive breast cancer in the National Institutes of Health-AARP Diet and Health Study cohort. J Natl Cancer Inst 2007; 99: 451.

26. Saxe GA, Rock CL, Wicha MS, et al. Diet and risk for breast cancer recurrence and survival. Breast Cancer Res Treat 1999; 53: 241-253.

27. Lynch BM, Neilson HK, Friedenreich CM. Physical activity and breast cancer prevention. Recent Results Cancer Res 2011; 186: 13-42.

28. Bauer SR, Hankinson SE, Bertone-Johnson ER, et al. Plasma vitamin D levels, menopause, and risk of breast cancer: dose-response meta-analysis of prospective studies. Medicine (Baltimore) 2013; 92: 123.

29. Bagnardi V, Rota M, Botteri E, et al. Light alcohol drinking and cancer: a meta-analysis. Ann Oncol 2013; 24: 301-308.

30. Key T, Appleby P, Barnes I, et al. Endogenous sex hormones and breast cancer in postmenopausal women: reanalysis of nine prospective studies. J Natl Cancer Inst 2002; 94: 606-616.

31. Eliassen AH, Missmer SA, Tworoger SS, et al. Endogenous steroid hormone concentrations and risk of breast cancer among premenopausal women. J Natl Cancer Inst 2006; 98: 1406-1415.

32. Kaaks R, Berrino F, Key T, et al. Serum sex steroids in premenopausal women and breast cancer risk within the European Prospective Investigation into Cancer and Nutrition (EPIC). J Natl Cancer Inst 2005; 97 : 755-765.

33. Fortner RT, Eliassen AH, Spiegelman D, et al. Premenopausal endogenous steroid hormones and breast cancer risk: results from the Nurses' Health Study II. Breast Cancer Res 2013; 15: R19.

34. Farhat GN, Cummings SR, Chlebowski RT, et al. Sex hormone levels and risks of estrogen receptor-negative and estrogen receptor-positive breast cancers. J Natl Cancer Inst 2011; 103: 562-570.

35. Goss PE, Ingle JN; NCIC CTG MAP.3 Study Investigators, et al. Exemestane for breast-cancer prevention in postmenopausal women. N Engl J Med 2011; 364: 2381-2391.

36. Cuzick J, Sestak I, Forbes JF, et al. Anastrozole for prevention of breast cancer in high-risk postmenopausal women (IBIS-II): an international, double-blind, randomised placebo controlled trial. Lancet 2014; 383: 1041-1048.
37. Visvanathan K, Hurley P, Bantug E, et al. Use of pharmacologic interventions for breast cancer risk reduction: American Society of Clinical Oncology clinical practice guideline. J Clin Oncol 2013; 31: 2942-2962.

38. Westhoff $\mathrm{CL}$. Breast cancer risk: perception versus reality. Contraception 1999; 59 (1 Suppl): 25S-28S.

39. Marchbanks PA, McDonald JA, Wilson HG, et al. Oral contraceptives and the risk of breast cancer. N Engl J Med 2002; 346: 2025-2032.

40. Collaborative Group on Hormonal Factors in Breast Cancer Lancet. Breast cancer and hormonal contraceptives: collaborative reanalysis of individual data on 53297 women with breast cancer and 100239 women without breast cancer from 54 epidemiological studies. Lancet 1996; 347: 1713-1727.

41. Hunter DJ, Colditz GA, Hankinson SE, et al. Oral contraceptive use and breast cancer: a prospective study of young women. Cancer Epidemiol Biomarkers Prev 2010; 19: 2496-2502.

42. Marchbanks PA, Curtis KM, Mandel MG, et al. Oral contraceptive formulation and risk of breast cancer. Contraception 2012; 85: 342-350.

43. Dinger J, Bardenheuer K, Minh TD. Levonorgestrel-releasing and copper intrauterine devices and the risk of breast cancer. Contraception 2011; 83: 211-217.

44. Prentice RL, Chlebowski RT, Stefanick ML, et al. Conjugated equine estrogens and breast cancer risk in the Women's Health Initiative clinical trial and observational study. Am J Epidemiol 2008; 167: 1407-1415.

45. Fournier A, Berrino F, Clavel-Chapelon F. Unequal risks for breast cancer associated with different hormone replacement therapies: results from the E3N cohort study. Breast Cancer Res Treat 2008; 107: 103-111.

46. Tamimi RM, Hankinson SE, Chen WY, et al. Combined estrogen and testosterone use and risk of breast cancer in postmenopausal women. Arch Intern Med 2006; 166: 1483-1489.

47. Banks E, Reeves G, Beral V, et al. Influence of personal characteristics of individual women on sensitivity and specificity of mammography in the Million Women Study: cohort study. BMJ 2004; 329: 477.

48. McTiernan A, Martin CF, Peck JD, et al. Women's Health Initiative Mammogram Density Study Investigators. Estrogen-plus-progestin use and mammographic density in postmenopausal women: Women's Health Initiative randomized trial. J Natl Cancer Inst 2005; 97: 1366-1376.

49. McTiernan A, Chlebowski RT, Martin C, et al. Conjugated equine estrogen influence on mammographic density in postmenopausal women in a substudy of the women's health initiative randomized trial. J Clin Oncol 2009; 27: 6135-6143.

50. Martin LJ, Minkin S, Boyd NF. Hormone therapy, mammographic density, and breast cancer risk. Maturitas 2009; 64: 20-26.

51. Chlebowski RT, Hendrix SL, Langer RD, et al. Influence of estrogen plus progestin on breast cancer and mammography in healthy postmenopausal women: the Women's Health Initiative Randomized Trial. JAMA 2003; 289: 3243-3253. 